\title{
Synthesis of silver nano particles and fabrication of aqueous Ag inks for inkjet printing
}

\author{
A. Kosmala, R. Wright, Q. Zhang*, and P. Kirby \\ Department of Materials, Cranfield University, Cranfield, Bedfordshire, MK43 0AL, UK \\ *Author for correspondence q.zhang@ cranfield.ac.uk
}

\begin{abstract}
The main problem in preparing stable and printable inks containing nanoparticles for inkjet printing is to overcome the strong agglomeration of the particles in dispersion medium. In this study, the silver particles with diameter around $50 \mathrm{~nm}$ were produced by a simple wet chemistry method. Stable aqueous printable inks were formulated by using the combination of a triblock copolymer and high intensity focused ultrasound (HIFU). Various factors that affect the ink stability, such as, copolymer content and time of HIFU treatment, were investigated. The ink containing $5 \mathrm{wt} \%$ silver has a viscosity of about $2 \mathrm{mPas}$ and surface tension $30 \mathrm{mN} / \mathrm{m}$ at $25{ }^{\circ} \mathrm{C}$, which meet inkjet printer requirements. Such inks have been successfully printed on $\mathrm{Al}_{2} \mathrm{O}_{3}$ ceramics and low-temperature co-fired ceramics (LTCC) and the printed films show low resistivity.
\end{abstract}

\section{Introduction}

There have been growing interest in the development of printed electronics in last few years because printed electronics offer alternatives to traditional silicon techniques and the potential for low cost, large area electronics for flexible displays [1-2], sensors [3-4] and polymer MEMS [5-6]. Printed electronics with various functional inks containing nanomaterials such as metallic nanoparticles, organic electronics molecules/polymers, carbon nanotubes, and ceramic nanoparticles have been expected to grow rapidly as a mass production process for new types of 
electronic equipments. The printing of metal inks, especially those containing silver nanoparticles, has been found to be a very powerful tool for direct patterning of electrically conductive interconnects in electronic devices [7-9]. The particle-based inks require powders with diameter less than $1 / 100$ the size of the nozzle diameter that is usually in a few or a few tens of micrometers, otherwise nozzle clogging would normally occur. Such a small size gives the particles unique properties which directly relate to their dimensions and to the fact that a large ratio of the atoms in the particle is in the surface of the particle. One of the advantages coming from the nano-size of material is a dramatic reduction in melting point. Films printed using such small particles can be annealed at lower temperatures to form conductive films of low resistance, and be potentially suitable for use in printed electronics [10-12]. However, generally speaking, nanoparticles tend to cluster and agglomerate if they are not prevented to do so. Particle agglomeration causes the system to change to the one with fewer but larger particles. Hence, it is necessary to modify the surface of the particles so that the agglomeration or clustering can be delayed.

One important aspect of inkjet printing technology is the physical properties of an ink, such as, viscosity and surface tension. Viscosity, to a certain extent, determines the formation of drops and in particular, the break-up and corresponding tail and surface tension is responsible for the spherical shape of the liquid drops emerging from a nozzle. It is possible to jet a wide variety of fluids with viscosities in the range of $2-30 \mathrm{mPa}$ s and surface tensions up to $60 \mathrm{mN} / \mathrm{m}$ [13].

Excellent dispersion of metal particles in the ink with a low viscosity and high resistance to sedimentation is essential to achieve printable fluid and assure its performance. Due to agglomeration of particles, the viscosity tends to increase during storage. Hence it is important to perform the viscosity measurement with storage time to obtain some information regarding the 
agglomeration. The good stability of an ink should stop particles from settling for at least one week at room temperature [13].

There are a few commonly used dispersion methods such as ultrasounds or flow impingement [13]. Different dispersants have been reported in literature to formulate stable $\mathrm{Ag}$ inks, for example, poly( $N$-vinyl-2-pyrrolidone (PVP) [14] and polyelectrolytes [15]. Most used solvents are toluene, alfa-terpineol [16-18]. However in the case of using organic solvents, it may cause the environmental problem by volatile organic compounds (VOCs) in realistic industrial applications. In the case of using aqueous medium [19-22], polyelectrolytes, such as, poly(acrylic acid) sodium salt [19], showed to be good capping agents and dispersants for Ag ink preparation. Because the synthesized Ag nanoparticles are agglomerated or clustered together, they must be separated into smaller particles for ink preparation. In many published work ball milling was used to break up the agglomerates, although it is an effective way, the beadcontamination of nanoparticle suspension might take place during milling process [23]. Bead breakage results in fragments which can not be removed from the nanoaprticles suspension and hence will contaminate the fluid.

Another way to formulate Ag inks is the use of metal-organic complexes as precursors (MOD inks) [24-26]. However, the disadvantage of this method is its high light sensitivity and high decomposition temperature.

In this work, very stable, water-based Ag inks were prepared, which is suitable for printing on ceramic substrates. The Ag nanoparticles prepared in our laboratory are strongly bound together through van der Waals force and therefore high energy is required in the dispersion process. High Intensity Frequency Ultrasound (HIFU), which has been used to breaking and deaggregating micro-sized particles into nano-sized ones and effectively forming nano suspensions 
[27], was used in this study to dispersing the Ag nanoparticles in water with the existence of dispersant Pluronic F127. As a "top-down" technique, and in comparison with conventional ultrasonic agitation and ball milling methods, HIFU introduces several novel aspects: (1) The high amplitudes (up to $10^{8} \mathrm{~Pa}$ ) and frequency $\left(\sim 10^{6} \mathrm{~Hz}\right)$ mean that as a beam propagates, it deforms, and can develop a "shock" wave front, i.e. the non-linear propagation effects; (2) Since the frequency of ultrasound is increased from $\sim 10^{4} \mathrm{~Hz}$ to $\sim 10^{6} \mathrm{~Hz}$, the threshold value of strong micro scale transient cavitations are significantly increased. Therefore, the immense temperatures and pressures and the extraordinary heating and cooling rates generated by cavitation bubble collapse could be alleviated. There's no distinct temperature increase at all [27]; (3) It avoids the possible contamination to the fluid due to bead breakage during ball milling and the prolonged ball milling process. The process of ball milling to disperse $\mathrm{Ag}$ nanoparticles in water needs a few tens of hours, in some cases, even days, while HIFU process needs only tens of minutes.

F127 is a triblock co-polymer consisting of poly(ethylene oxide) (PEO)-poly(propylene oxide) segments arranged in an ABA structure. The PEO block is hydrophilic, and the PPO block hydrophobic. F127 micelles has been demonstrated to enable encapsulating hydrophobic Ag nanoparticles inside their PPO cores and simutaneously the surface of the encapsulated nanoparticles is intrinsically covered by a layer of free PEO chains, which enable the $\mathrm{Ag}$ particles to be colloidally stable [28]. The combination of HIFU technique and F127 triblock copolymer is expected to allow us to produce long-term stable Ag inks, suitable for Ag inkjet printing. The effects of copolymer concentration, silver content and time of HIFU treatment on the rheological behaviour and size distribution were studied and an investigation on the sedimentation rate of the ink was also performed in this study. 


\section{Experimental}

Ag nanoparticles were synthesized by a new approach. All chemicals were used as purchased. Silver nitrate $(2 \mathrm{~g})$ used as Ag precursor and surfactant dioctyl sodium sulfosucinate (AOT, 4g) as a capping agent were separately dissolved in water, forming $0.03 \mathrm{M}$ and $0.02 \mathrm{M}$ solutions respectively and then two solutions were mixed by adding the surfactant solution to the metal salt one. The mixture was kept stirring to form a foam. $40 \mathrm{ml}$ of hydrazine, $\mathrm{N}_{2} \mathrm{H}_{4} \mathrm{OH}(1.5$ $\mathrm{mM}$ ) was then added to the solution. To complete the reaction $40 \mathrm{ml}$ of $0.1 \mathrm{M} \mathrm{NaOH}$ aqueous solution was added to precipitate out Ag naoparticles. The obtained particles were then washed with water and dried at room temperature. The synthesized Ag nanoparticles were firstly suspended in water with wt $\%$ of Pluronic F127 ranging from $0.3 \%-1.5 \%$. The suspensions to be treated by HIFU were contained in a sample tube/beaker which was fixed above the shock wave generator (JK-06) developed by Nanjing University of Aeronautics and Astronautics. The frequency of ultrasound is set at $\sim 75 \mathrm{kHz}$, and the peak intensity of shock wave varies between 0 80 MPa. The treated time was varied between 10 and $90 \mathrm{~min}$.

Inkjet printing was carried out with a DoD Fujifilm Dimatix DMP-2831 ink jet printer. The inks were printed on two different substrates, alumina $\left(\mathrm{Al}_{2} \mathrm{O}_{3}\right)(480 \mu \mathrm{m})$, and low temperature co-fired ceramic (LTCC) $(250 \mu \mathrm{m})$ tapes. Disposable cartridges (DMC-11610) used in this work are equipped with 16 silicon nozzles located at $254 \mu \mathrm{m}$ spacing; the orifice size of each nozzle is approximately $21 \mu \mathrm{m}$. The ceramics play an important role in electronics miniaturization and have potential to become a mass production technology for non-silicon Microsystems. Using ceramic tapes it is possible to integrate small passive components on ceramic substrates in 3-D that gives considerable possibilities for electronic miniaturization. 
The size and morphology of silver nanoparticles and printed patterns were determined using scanning electron microscopy (SEM). X-ray diffraction (XRD) was used to determine the crystal structure of particles. Particle size distribution was determined by nanoparticle tracking analysis (NTA) (LM20, Nanosight). The rheological behaviour including stability studies was conducted using CVO rheometer (Bohlin instrument). The surface energy measurement was performed on a contact angle system (OCA-Dataphysics). Continuous lines with $300 \mu \mathrm{m}$ wide, $4.5 \mathrm{~mm}$ long and $2 \mu \mathrm{m}$ thick were heated at $350{ }^{\circ} \mathrm{C}$ for $1 \mathrm{~min}$. After cooling down to room temperature the conductivity measurements were performed using a four point probe technique. The probes have been put in the four corners of the printed track and measurement of resistance has been taken three times. The electrical resistivity of the printed lines was then calculated.

\section{Results and discussion}

Silver nanoparticles were produced in water by reduction of silver nitrate with hydrazine in the presence of AOT. The $\mathrm{Ag}^{+}$ions are firstly converted into silver atoms. The silver atoms then precipitate to form silver nanoparticles with AOT absorbed on the surface of the silver nanoparticles. The color of the solution gradually changed from light brown to brown and even to dark brown. SEM micrograph and XRD pattern of Ag nanoparticles are shown in Fig. 1. The primary Ag grains are spherical with about $20 \mathrm{~nm}$ in diameter (Fig 1a) and the XRD pattern of the silver nanoparticles obtained by $\mathrm{Ag}^{+}$reduction with hydrazine was shown in Fig $1 \mathrm{~b}$. The $\mathrm{XRD}$ pattern of the $\mathrm{Ag}$ shows that five diffraction peaks at $2 \theta$ of $38.2^{\circ}, 44.4^{\circ}, 64.5^{\circ}, 77.5^{\circ}$ and $81.6^{\circ}$ respectively could be indexed as (111), (200), (220), (311) and (222) Bragg's reflections (JCPDS file No. 04-0783) of the face-centred cubic (fcc) structure of silver. The particle size could be calculated from the Ag (111) diffraction line using Scherrer's formula [29], $\mathrm{t}=\mathrm{k} \lambda / \beta$ 
$\cos \theta$, where $t$ was the mean dimension of the crystallites, $\beta$ was the full width at half maximum of the diffraction peak, $\theta$ was the diffraction angle, $\lambda$ was the wave length of $\mathrm{Cu} \mathrm{K}$ radiation $(0.1540 \mathrm{~nm})$, and $\mathrm{k}$ was a constant (0.89). The calculated size of silver nanoparticle protected by AOT was about $20 \mathrm{~nm}$, which is a good agreement with the observation by SEM.

Particle size distribution analysis shows that the agglomerated particle sizes of the $\mathrm{Ag}$ nanoparticles are in the range of 10-300 nm, with the majority of particles larger than $120 \mathrm{~nm}$ as shown in Fig. 2.

The aqueous foam was formed as a result of the mixture of AOT solution with $\mathrm{AgNO}_{3}$ solution. $\mathrm{Ag}^{+}$ions were bonded electrostatically with AOT molecules at the air bubble - solution interface as shown in Fig. 3. The silver ions were reduced in the foam by hydrazine resulting in the formation of silver nanoparticles. The air bubble - solution interface provides a high concentration of nucleation centres at which silver particles grow. The surface therefore acts as a good template for crystallization.

The primary nanoparticles strongly attract each other through physical forces due to large surface area, immediately forming larger particles.

HIFU has been used to assist deagglomeration and F127 to stabilize the nanoparticles in water in order to obtain stable inks or nanofluids. Fig. Fig. 4 shows the influence of dispersant concentration on the particle size distribution of $1 \mathrm{wt} \% \mathrm{Ag}$ aqueous suspension, at different HIFU times. It can be seen that the average particle size is reduced with the increase of dispersant (F127) amount and reaches the minimum at the concentration of $0.6 \mathrm{wt} \%$ at any given HIFU time. However, at a given dispersant concentration the average silver particle size firstly decreases with the time of HIFU treatment up to $60 \mathrm{~min}$, and then increases to larger value at HIFU treatment for 90 min. The minimum particle size was obtained in a silver suspension with 
$0.6 \mathrm{wt} \%$ F127 after 60 min HIFU treatment. Increasing the time of HIFU treatment leads to finer particles and thus increases the surface area, which needs more dispersant to cover the fresh surface. Therefore the amount of dispersant at tested F127 wt $\%$ may not be enough therefore leading to the reagglomeration. It is known that the adhesive forces of nanofine particles increase rapidly and become the dominant factor.

Fig.5 illustrates the change of average particle size against HIFU treatment time for different $\mathrm{Ag}$ contents at a given dispersant concentration. With the increase of the HIFU treatment time up to $60 \mathrm{~min}$, the particle size decreases in each sample. The less Ag content, the less particle size can be obtained under HIFU treatment. Although the minimum size is possible to obtain in the suspension with $5 \mathrm{wt} \% \mathrm{Ag}$ (60 min HIFU treatment), the increase of silver content up to $10 \mathrm{wt} \%$ in a suspension slows down the disaggregation process. The HIFU treatment time required to reach the minimum size increases with the increase of $\mathrm{Ag}$ content. Therefore, the further work in this study focuses on the analysis of the Ag fluid with solid loading between 1 and $5 \mathrm{wt} \%$.

Insufficient amount of dispersant would result in the flocculation of some particles while excessive amount of dispersant would increase the viscosity, either of these is causing destabilisation of the suspension. Therefore it is important to determine the optimal amount of dispersant in a particle suspension. Fig. 6 shows the change of viscosity against the concentration of dispersant at $1 \mathrm{wt} \% \mathrm{Ag}$ concentration suspension. The HIFU treatment time is varied from 10 to $90 \mathrm{~min}$ for each sample. As can be seen, the viscosity values at any given HIFU treatment time decrease gradually with increasing concentration of dispersant and reach a minimum at the concentration of $0.6 \mathrm{wt} \%$. Further addition of dispersant raises the viscosity of the suspension. This specific amount of copolymer is related to the size and the surface area of the particle to be 
covered: smaller particle size means higher surface area and hence higher amount of dispersant is needed. These results shown in Fig. 4 \& 6 imply that the amount of F127 required for monolayer coverage and the amount required for reaching the minimum viscosity correlate very well.

Fig. 7 presents the ink rheological behaviour. It can be seen, as expected, that the viscosity rises with increasing silver content in a fluid. That is due to the increase of the interaction between silver particles when solid content increases. The viscosity value for $1 \mathrm{wt} \%$ of silver in ink is $1.6 \mathrm{mPa}$ s and it rises up to $2.1 \mathrm{mPa}$ s when silver loading increases to $5 \mathrm{wt} \%$.

Fig. 8 shows the SEM picture of Ag nanoparticles in the nanofluid treated with HIFU for $60 \mathrm{~min}$. In this nanofluid, Ag content was fixed at $5 \mathrm{wt} \%$. It was found that Ag nanoaprticles were shown to be homogeneously distributed in the nanofluid. Without any physical treatment, Ag nanoparticles were highly agglomerated as shown in Fig 8 (a) .

To evaluate the stability of nanofluids treated by HIFU with the presence of F127 copolymer, the viscosity of nanofluids, containing $5 \mathrm{wt} \%$ Ag nanoparticles, $0.6 \mathrm{wt} \%$ dispersant and treated by HIFU for 60 min, was measured against the time over 20 days (Fig. 9). It was found that there was almost no viscosity change up to 10 days and after this time, viscosity linearly but slowly increased with the time. The increase of viscosity from $2.1 \mathrm{mPa}$ s in Day one to $2.3 \mathrm{mPa}$ s in Day 20 was due to the agglomeration and the formation of larger particle clusters. However, no sedimentation was observed up to 20 days. The formed large particle clusters due to the agglomeration could be broken and re-dispersed in the water if HIFU treatment is re-used and the rhoelogical properties of the dispersion can resume to the inital values. We are able to load $\mathrm{Ag}$ content in our inks up to $25 \mathrm{wt} \%$, but the particles in the inks form big agglomerates within a few days and subsequently the inks block the printing head. The work is ongoing to 
optimise the ratio of F127 and Ag content and HIFU treatment time for high Ag loading (>25 wt $\%$ ) fluids.

Stable nanofluid is one of the key elements in inkjet printing. Three kinds of forces act on the suspended particles in the liquid, gravitational force, tending to settle or rise particles depending on their density relative to the solvent; a viscous drag force which arises as a resistance to motion, and the kinetic energy of particles which causes Brownian motion.

For spherical particle of radius $r$ the viscous drag force must be equal to the gravitational force thus the settling velocity, $V$, the viscous drag force is given by:

$$
F_{\text {drag }}=6 \pi r V \eta=4 \pi r^{3} g\left(\rho_{p}-\rho_{L}\right) / 3=F_{\text {gravity }}
$$

where, $\eta$ is the viscosity of the water, $\rho_{P}$ is the densities of the particles and $\rho_{L}$ is the density of the medium and $\mathrm{g}$ is the acceleration rate of the gravity.

Clearly, from those two forces acting on small particles, it will take a very long time to settle the particles and they will be stable almost endlessly.

In fact, the third force caused by Brownian motion, together with the attractive and repulsive forces between particles determined the stability of the nanofluids. The speed of the Brownian motion can be characterised by mean displacement and can be expressed with the equation:

$$
x^{2}=2 \cdot D \cdot t
$$

where $D$ is the Einstein's Brownian diffusion coefficient and $t$ is the diffusion time.

It is possible to express the diffusion coefficient with Einstein equation:

$$
D=\frac{k T}{3 \pi \eta r}
$$


Where $k$ is the Boltzman constant, $T$ is temperature, $\eta$ is viscosity of the medium and $r$ is the particle size.

Based on Eq. (1) and (2), it can be said that displacement caused by Brownian motion increases with decreasing particles size and hence the nanoparticles tend to move around approaching other nanoparticles. The attractive forces between the particles will lead to their collision and cause the growth of large aggregates, which will consequently settle them out as, with particle growth, the gravitational forces start to dominate particle's behaviour [30].

Although the van der Waals forces acting between molecules will always act to coagulate dispersed colloids, it is possible to generate an opposing repulsive force of comparable strength. As smaller particles have higher surface energy that increases the possibility of the agglomeration of nanoparticles, they must be stabilized by surfactant or capping reagent in their dispersed medium [30].

The prepared Ag ink has low viscosity ( 2 $\mathrm{mPa}$ s) and desirable surface energy, 30 $\mathrm{mN} / \mathrm{m}$, that is lower than the surface energy of substrates $\left(54 \mathrm{mN} / \mathrm{m}\right.$ for $\mathrm{Al}_{2} \mathrm{O}_{3}$ and $52 \mathrm{mN} / \mathrm{m}$ for LTCC), hence it ensures good wetting of the substrates by the ink; also this surface energy of the ink is lower than the inkjet printer requirement for surface energy $(\max .60 \mathrm{mN} / \mathrm{m})$ in order to jet droplets.

Fig. 10 shows optical microscope pictures of printed ink on a LTCC substrate. After printing, the solvent was evaporated by heating. Direct physical contact between the particles occurred after the organic reagents were burnt off, beginning to show neck formation [21, 31]. As Ag loading in the ink is low, the multilayer printing approach helps build up dense and conductive films. The film consisted of 60 layers showed a reasonably low resistivity. Fig. 11 shows the SEM pictures of printed $\mathrm{Ag}$ layers on $\mathrm{Al}_{2} \mathrm{O}_{3}$. The film in Fig 11 (a) consisted of 60 
layers and was fired together at $350{ }^{\circ} \mathrm{C}$ for $60 \mathrm{~min}$. The film in Fig 11 (b) also consisted of 60 layers, but fired at $350^{\circ} \mathrm{C}$ for $20 \mathrm{~min}$. after the deposition of each 20 layers. The resistivity of the film with layers fired together showed a value of $4 \pm 0.1 * 10^{-7} \Omega \mathrm{m}$, while the film fired after the deposition of each 20 layers showed a $2 \pm 0.1 * 10^{-7} \Omega \mathrm{m}$. As can be seen in Fig. 11, the sintering between printed layers improves final structure of the film in terms of its density which is directly linked to the better conductivity. The difference between grain structures might be due to easier evaporation of solvent that takes place while printing in steps. Printing high number of layers on top of each other results in a trapping of the water and surfactant molecules in a formed structure which eventually generates pores, hence reduces the density of the film, and thus lowers the conductivity of printed features.

\section{Conclusion}

Silver nanoparticles have been prepared through the reduction of silver ions by hydrazine in the presence of AOT. This is a simple, environmental-friendly and cost effective process. Particles are agglomerated and the average size of primary particles is about $20 \mathrm{~nm}$. A stable Ag ink was prepared by dispersing Ag nanoparticles in water with the presence of a triblock copolymer, PEO-PPO-PEO, and then being treated with high intensity focus ultrasound. The investigation of HIFU treatment showed that the size of Ag agglomerates was reduced down to $\sim 50 \mathrm{~nm}$ from $200 \mathrm{~nm}$ untreated. The viscosity of the nanofluid was reduced after sonication indicating that the HIFU treatment could help separating the agglomerates and relatively stable inks indicated the high efficiency of the copolymer in stabilizing Ag nanoparticles in water. The printed films on $\mathrm{Al}_{2} \mathrm{O}_{3}$ tapes and LTCC substrates showed the very low resistivity. 


\section{Acknowledgments}

Authors would like to thank the European Project (FP7) "MULTILAYER: a large scale production of micro devices via new rolled multi material layered 3D shaping technology", for the financial support with the reference number: FP7-NMP4-2007-214122. We thank Swerea IVF for providing us LTCC tapes and $\mathrm{Al}_{2} \mathrm{O}_{3}$ ceramics. We thank Omnisys Instruments for the design of the printed patterns and Altatech for performing the printing on $\mathrm{Al}_{2} \mathrm{O}_{3}$. We thank EFPG, France for performing contact angle and surface energy measurements.

\section{References}

[1] J.W. Kang, W.I. Jeong, J.J. Kim, H.K. Kim, D.G. Kim and G.H. Lee, High-performance flexible organic light-emitting diodes using amorphous indium zinc oxide anode. Electrochem. Solid-State Lett. 10, 75, (2007).

[2] L. Hou, F. Huang, W. Zeng, J. Peng and Y. Cao, High-efficiency inverted top-emitting polymer light-emitting diodes. Appl. Phys. Lett. 87, (2005).

[3] V. Shamanna, S. Das, Z. Celik-Butler and K. L. Lawrence, Micromachined integrated pressure-thermal sensors on flexible substrates. J. Micromech. Microeng. 16, (2006).

[4] F. Jiang, G. B. Lee, Y. C. Tai and C. M. Ho, A flexible micromachine-based shear-stress sensor array and its application to separation-point detection. Sensor Actuators A 79, 194, (2000).

[5] S.A. Day, D.P. Butler and Z. Celik-Bulter, Micromachined infrared bolometers on flexible polyimide substrates. Sensors Actuators A 118, 49, (2005). 
[6] S. Tung, S.R. Witherspoon, L. A. Roe, A. Silano, D. P. Maynard and N. Ferraro, AMEMSbased flexible sensor and actuator system for space inflatable structures. Smart Mater. Struct. 10, 1230, (2001).

[7] K. J. Lee, B. H. Jun, T. H. Kim and J. Joung, Direct synthesis and inkjetting of silver nanocrystals toward printed electronics. Nanotechnology 17, 2424, (2006).

[8] D. Kim, S. Jeong, B. K. Park and J. Moon, Direct writing of silver conductive patterns: Improvement of film morphology and conductance by controlling solvent compositions. Appl. Phys. Lett. 89, 264101, (2006).

[9] T.H.J. Van Osch, J. Perelaer, A.W.M. De Laat and U. S. Schubert, Inkjet printing of narrow conductive tracks on untreated polymeric substrates. Adv. Mater. 20, 343, (2008).

[10] G. L. Allen, R. A. Bayles, W. W. Gile, and W. A. Jesser, Small particle melting of pure metals. Thin Solid Films 144, 297, (1986).

[11] P. Buffat and J-P. Borel, Size effect on the melting temperature of gold particles. Phys. Rev. A 13, 2287, (1976).

[12] Q. Jiang, S. Zhang, and M. Zhao, Size-dependent melting point of noble metals. Mater. Chem. Phys. 82, 225, (2003).

[13] S. Magdassi, The chemistry of silver ink, World Scientific Publishing, Singapure (2010).

[14] E. Tamjid and B. H. Guenther, Rheological and sedimentation behaviour of nanosilver colloids for inkjet printing. Int. J. Nanomanufacturing 5, 383, (2010).

[15] A. B. R. Mayer, Colloidal metal nanoparticles dispersed in amphiphilic polymers. Polym. Adv. Technol. 12, 96, (2001).

[16] P.j. Smith, D.Shin, J.E. Stringer, B.Derby and N. Reis, Direct ink-jet printing and low temperature conversion of conductive silver patterns. J.Mater.Sci. 41, 4153, (2006). 
[17] S. Fuller, E. J. Wilhelm and J. M. Jacobson, Ink-jet printed nanoparticle microelectromechanical systems. J. Microelectromech. Syst. 11, 54, (2002).

[18] J. Perelaer, Berend-Jan de Gans, and U. S. Schubert, Ink-jet printing and microwave sintering of conductive silver tracks. Adv. Mater. 18, 2101, (2006).

[19] S. Jeong, H. C. Song, W. W. Lee, Y. Choi, and B. H. Ryu, Preparation of aqueous Ag Ink with long-term dispersion stability and its inkjet printing for fabricating conductive tracks on a polyimide film. J. Appl. Phys. 108, 102805, (2010).

[20] S. Magdassi, A. Bassa, Y. Vinetsky, and A. Kamyshny, Silver nanoparticles as pigments for water-based ink-jet inks. Chem. Mater. 15, 2208, (2003).

[21] J. Perelaer, A. W. M. de Laat, C. E. Hendriks, and U. S. Schubert, Inkjet-printed silver tracks: low temperature curing and thermal stability investigation. J. Mater. Chem. 18, 3209, (2008).

[22] J-W Park and S-G Bae, Thermal behavior of direct-printed lines of silver nanoparticles. Scripta Materialia, 55, 1139, (2006).

[23] (a) H. Yoden, J.Itoh, Effect of small size beads on dispersion of nanometer-sized silica particle by wet beads mill process. J.Soc.Powder Technol.Jpn, 41, 457, (2004); (b) M.Inkyo, T.Tahara, T. Iwaki, F Iskandar, C.J. Hogan Jr. and K. Okuyama ${ }^{\mathrm{c}}$, Experimental investigation of nanoparticle dispersion by beads milling with centrifugal bead separation. Journal of Colloid and Interface Science, 304, 535, (2006).

[24] K. F. Teng, R. Vest, Liquid Ink Jet Printing with MOD Inks for Hybrid Microcircuits.IEEE Transactions on components, hybrids, and manufacturing technology, 12, 545, (1987). 
[25] F. Jahn, T.Blaudeck, R. Reinhard, Inkjet Printing of Conductive Silver Patterns by Using the First Aqueous Particle-Free MOD Ink without Additional Stabilizing Ligands. Chem. Mater. 22, 3067, (2010).

[26] A. L. Dearden' P.J. Smith, D-Y Shin, Nuno Reis, Brian Derby, Paul O'Brien, A Low Curing Temperature Silver Ink for Use in Ink-Jet Printing and Subsequent Production of Conductive Tracks. Macromol.Rapid Commun. 26, 315, (2005).

[27] W. Wang, Q. Zhang, Z. Liu, Z. Libor, Highly efficient size reduction of nanoparticles by the hock wave method. Functional Materials Letters, 3, 299, (2010).

[28] H. Tan, J. M. Xue, B. Shuter, X. Li and J. Wang, Synthesis of PEOlated $\mathrm{Fe}_{3} \mathrm{O}_{4} @ \mathrm{SiO}_{2}$ Nanoparticles via Bioinspired Silification for Magnetic Resonance Imaging. Adv. Funct. Mater. 20, 722, (2010).

[29] S.C. Chanda, A. Manna, V. Vijayan, K. Nayak Pranaba, M. Ashok, H.N. Acharya, PIXE \&amp; XRD analysis of nanocrystals of $\mathrm{Fe}, \mathrm{Ni}$ and $\mathrm{Fe}_{2} \mathrm{O}_{3}$. Mater. Lett. 61, 5059, (2007).

[30] W. Russel, D. Saville, W. Schowalter, Colloidal dispersion, Cambridge University Press, Cambridge, (1989).

[31] S.Sivaramakrishnan, P-J Chia, Y-C Yeo, L-L Chua and P. K.-H. Ho, Controlled insulatorto-metal transformation in printable polymer composites with nanometal clusters. Nat. Mater. 6, 149, (2007). 
Figure captions

Fig.1 SEM (a) and XRD (b) of silver particles

Fig 2 Size distribution of silver particles (NTA)

Fig. 3 Schematic diagram of silver particle synthesis mechanism

Fig. 4 Effect of different amount of copolymer into $1 \mathrm{wt} \%$ of Ag suspension on the particles size at different HIFU treatment time

Fig. 5 Particle size as a function of HIFU treatment time at different solid loading

Fig. 6 Effect of copolymer concentration on the viscosity of $1 \%$ Ag aqueous suspension at different HIFU treatment time

Fig. 7 Rheological behaviour of ink with different silver content. The ratio of Ag: F127 is fixed at 1:0.6 by weight.

Fig. 8 SEM of silver particles dispersed in water a) before and b) after HIFU treatment

Fig. 9 Viscosity change of nanofluid treated by HIFU in the presence of F127 against time

Fig. 10 Printed Ag lines on LTCC

Fig. 11 SEM pictures of cross section of the sintered silver on $\mathrm{Al}_{2} \mathrm{O}_{3}$, printed in one go (a) and printed in steps with heating between the layers (b) 

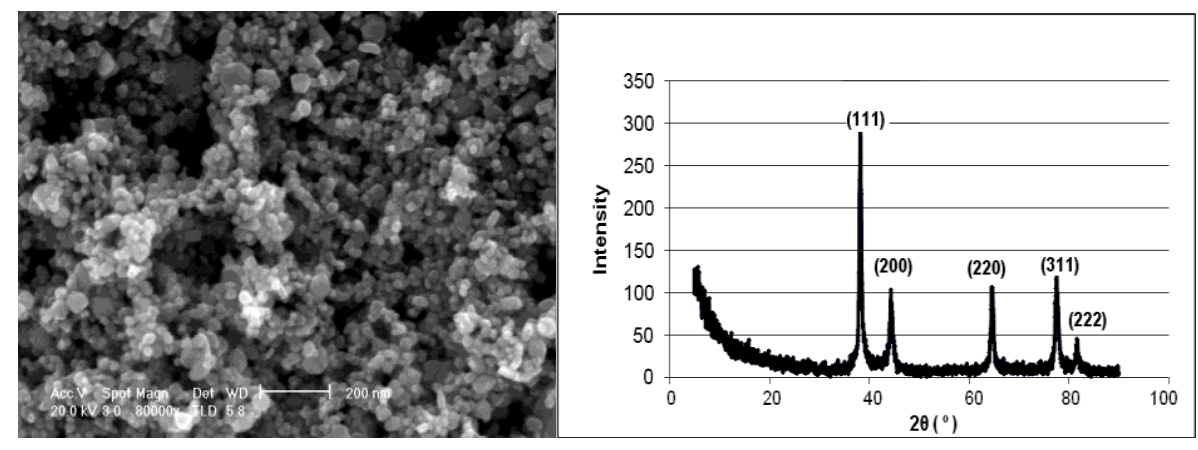

Fig 1

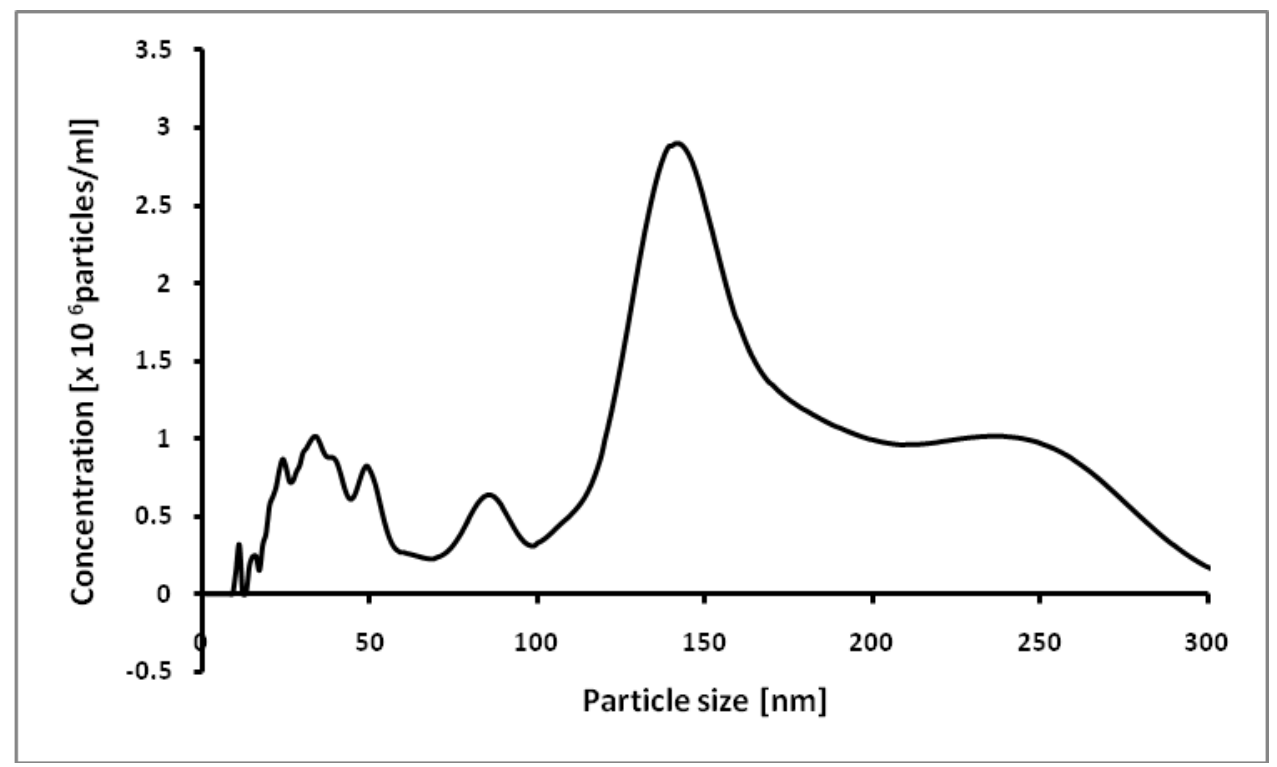

Fig. 2

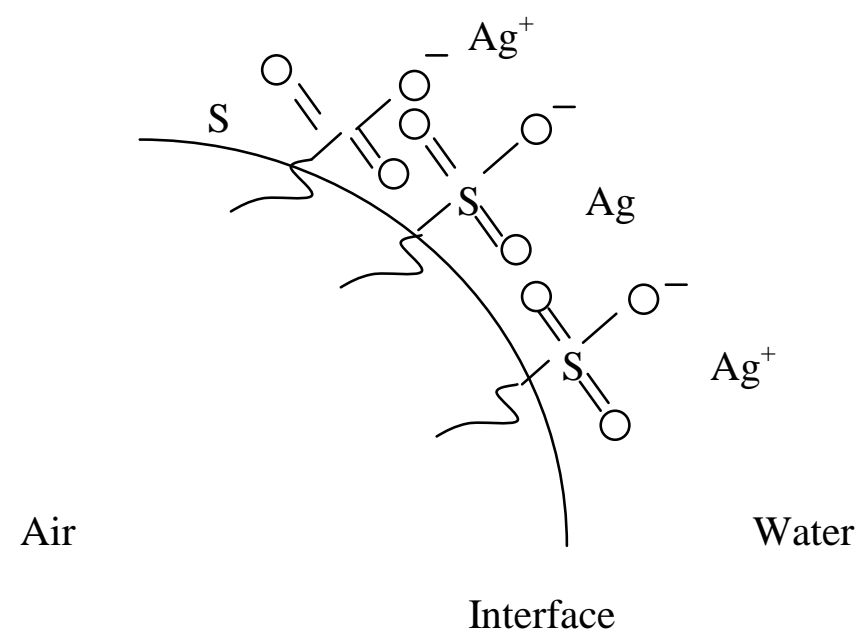

Fig. 3 


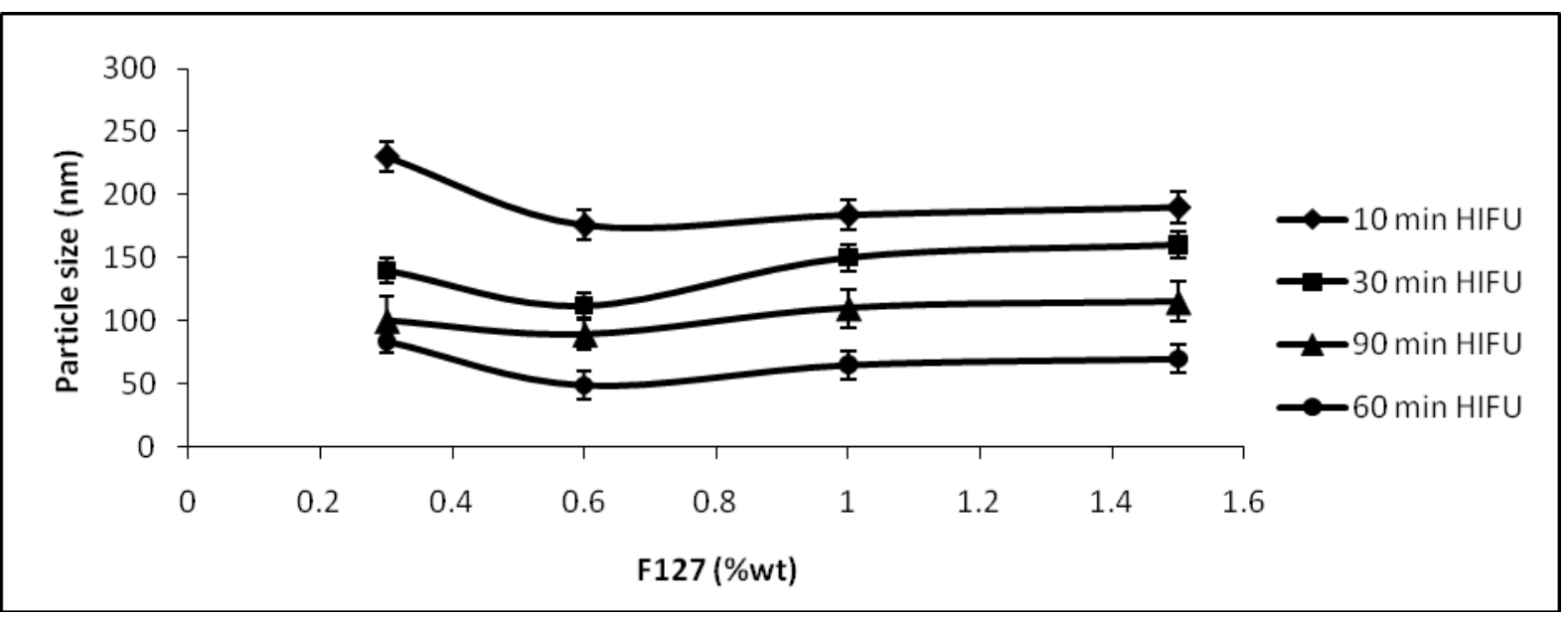

Fig. 4

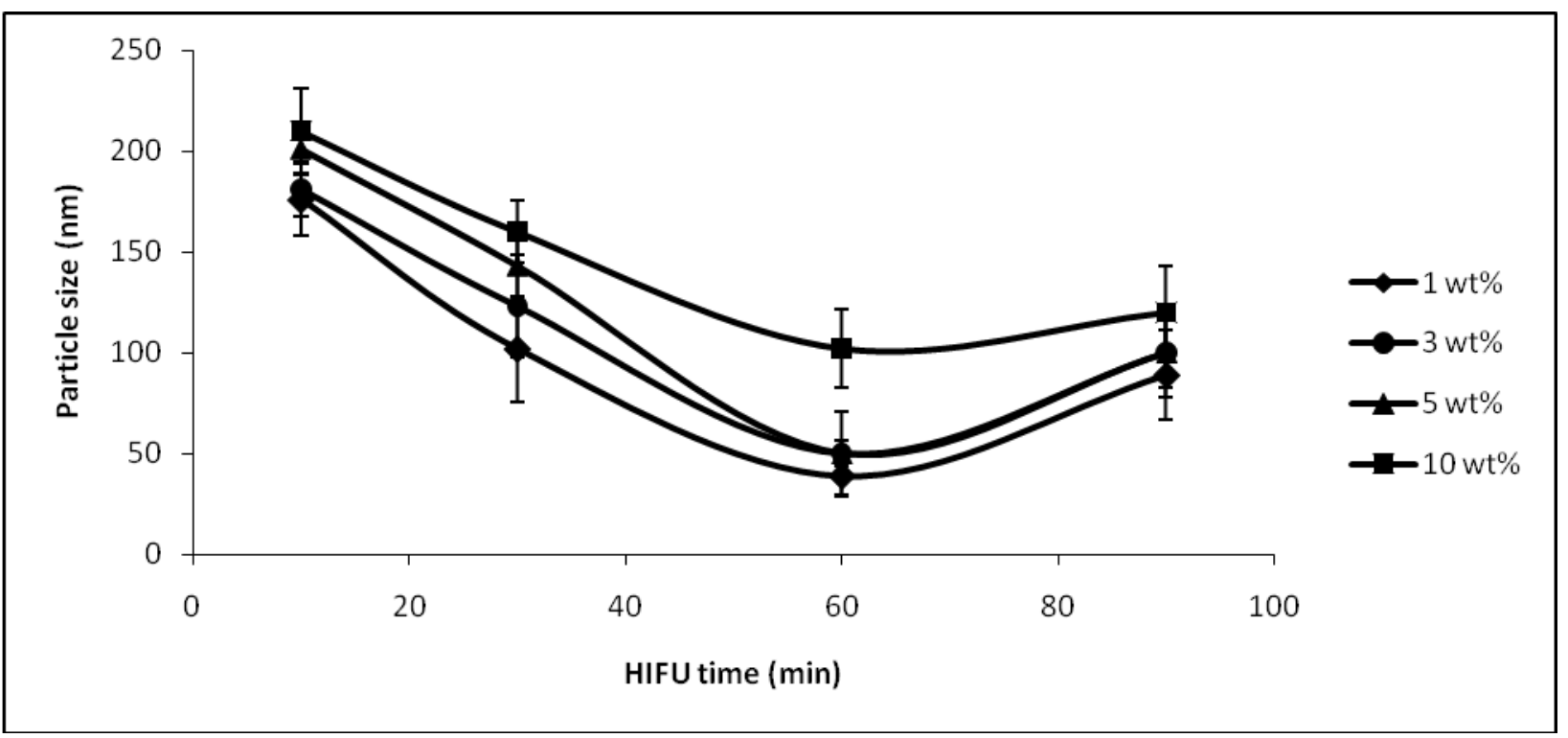

Fig.5 


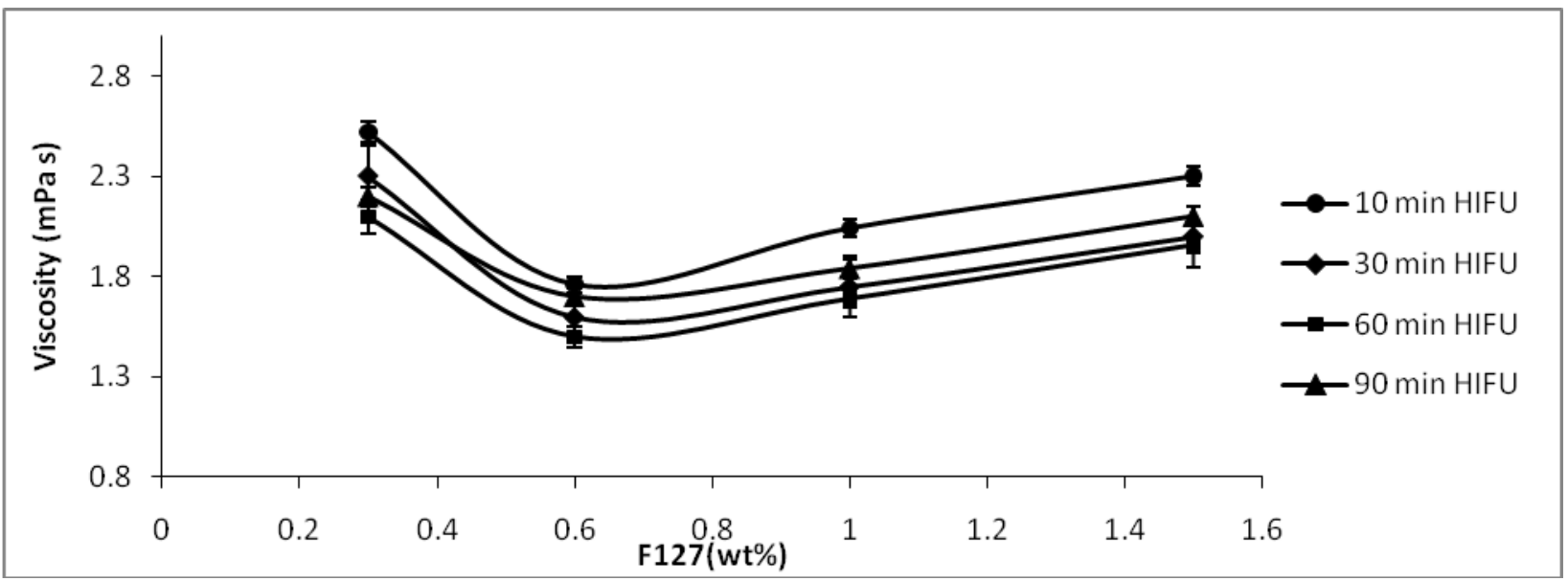

Fig. 6

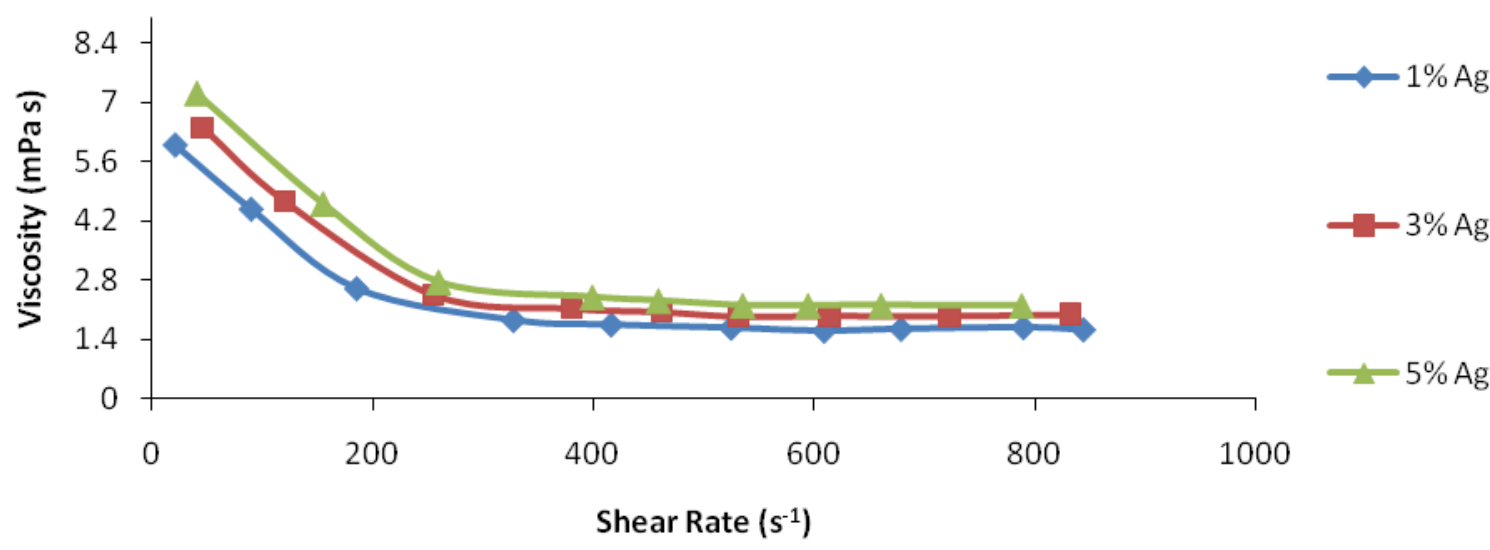

Fig. 7

a)

b)
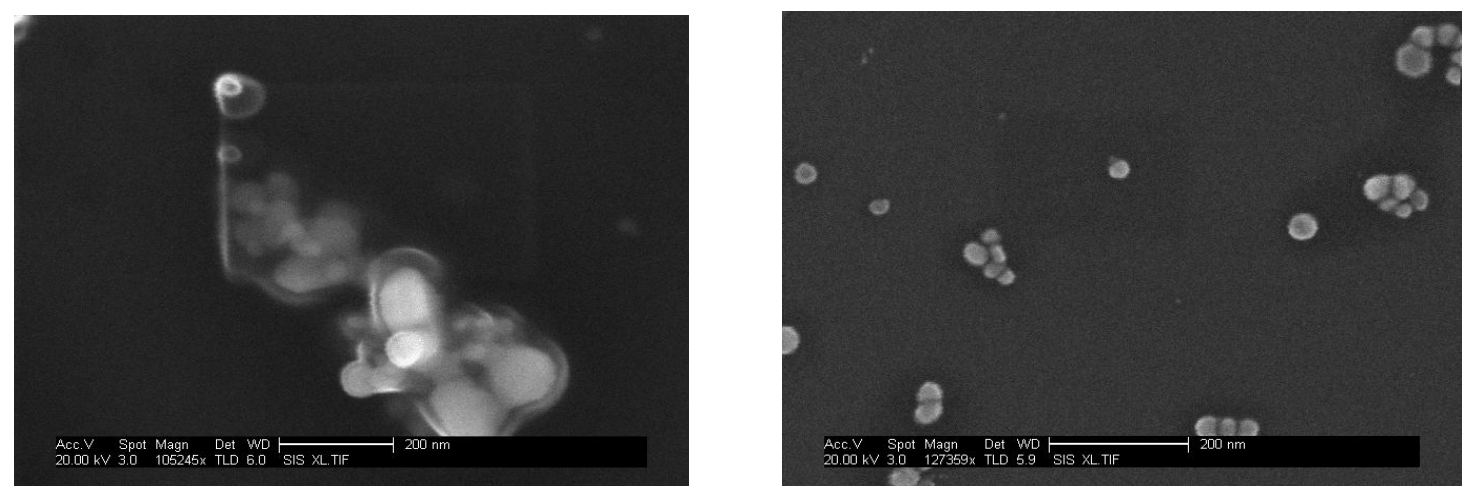

Fig.8 


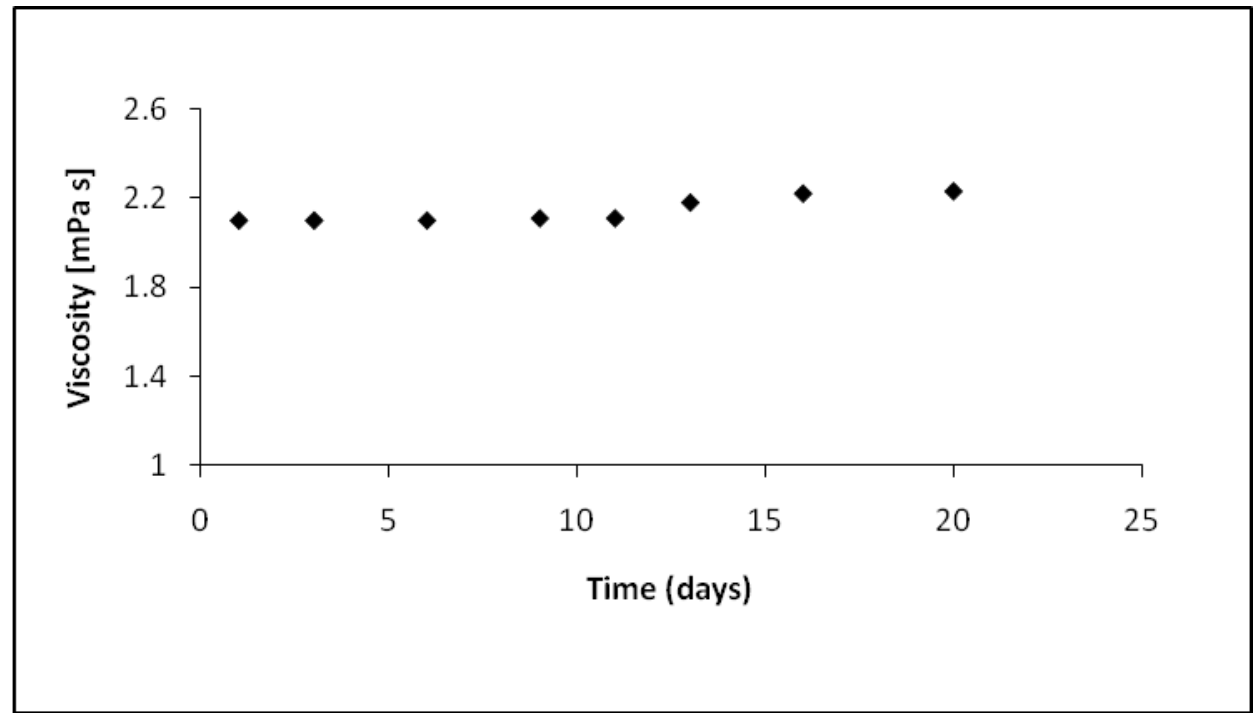

Fig. 9

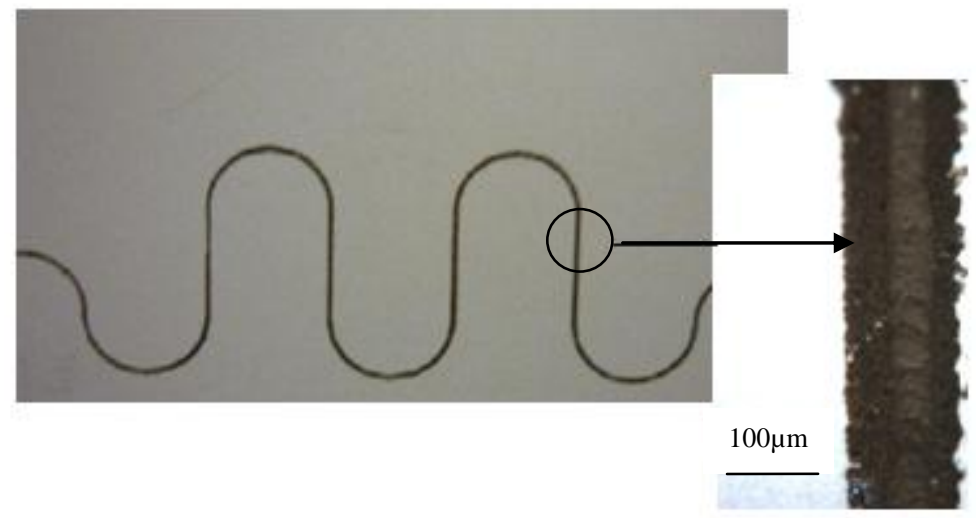

Fig. 10

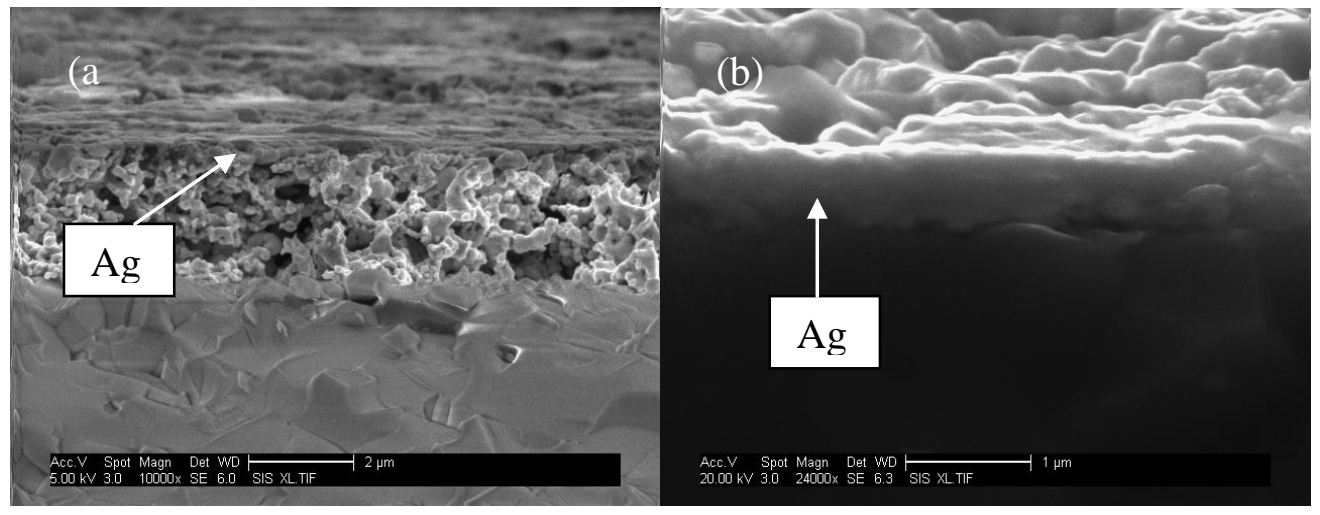

Fig. 11 